\title{
Endovenous laser ablation versus mechanochemical ablation with ClariVein in the management of superficial venous insufficiency (LAMA trial): study protocol for a randomised controlled trial
}

\author{
Clement C. M. Leung ${ }^{*}$, Daniel Carradice, Tom Wallace and lan C. Chetter
}

\begin{abstract}
Background: Endovenous thermal techniques, such as endovenous laser ablation (EVLA), are the recommended treatment for truncal varicose veins. However, a disadvantage of thermal techniques is that it requires the administration of tumescent anaesthesia, which can be uncomfortable. Non-thermal, non-tumescent techniques, such as mechanochemical ablation (MOCA) have potential benefits. MOCA combines physical damage to endothelium using a rotating wire, with the infusion of a liquid sclerosant. Preliminary experiences with MOCA showed good results and less post-procedural pain.

Methods/Design: The Laser Ablation versus Mechanochemical Ablation (LAMA) trial is a single-centre randomised controlled trial in which 140 patients will be randomly allocated to EVLA or MOCA. All patients with primary truncal superficial venous insufficiency (SVI) who meet the eligibility criteria will be invited to participate in this trial. The primary outcomes are intra-procedural pain and technical efficacy at 1 year, defined as complete occlusion of target vein segment and assessed using duplex ultrasound. Secondary outcomes are post-procedural pain, analgesia use, procedure time, clinical severity, generic and disease-specific quality of life, bruising, complications, satisfaction, cosmesis, time taken to return to daily activities and/or work, and cost-effectiveness analysis following EVLA or MOCA. Both groups will be evaluated on an intention-to-treat basis.

Discussion: The aim of the LAMA trial is to establish whether MOCA is superior to the current first-line treatment, EVLA. The two main hypotheses are that MOCA may cause less initial pain and disability allowing a more acceptable treatment with an enhanced recovery. The second hypothesis is that this may come at a cost of decreased efficacy, which may lead to increased recurrence and affect longer term quality of life, increasing the requirement for secondary procedures.
\end{abstract}

Trial registration: ClinicalTrials.gov identifier: NCT02627846, registered 8 December 2015 EudraCT number: 2015-000730-30

REC ref: $15 / \mathrm{YH} / 0207$

R\&D ref: R1788

Keywords: Mechanochemical ablation, ClariVein', MOCA, Endovenous laser ablation, EVLA, Superficial venous insufficiency, Varicose vein, Therapy, Treatment, Outcome

(Continued on next page)

* Correspondence: clement.leung@nhs.net

Academic Vascular Unit, Hull York Medical School, University of Hull, Hull HU3 2JZ, UK 


\begin{abstract}
(Continued from previous page)
Abbreviations: AASV, Anterior accessory saphenous vein; $A E$, Adverse event; $A R$, Adverse reaction; $A W Q$, Aberdeen Varicose Vein Questionnaire; CEAP, Clinical severity, Etiology, Anatomy, Pathophysiology; CIVIQ-20, Chronic Venous disease quality-of-life Questionnaire; CRF, Case report form; CTIMP, Clinical Trial of an Investigational Medicinal Product; DSUR, Development Safety Update Report; DUS, Duplex ultrasound scan; DVT, Deep vein thrombosis; EQ5D, EuroQol 5domain utility index; EVLA, Endovenous laser ablation; EVTA, Endovenous thermal ablation; GA, General anaesthesia; GSV, Great saphenous vein; IMP, Investigational medicinal product; LA, Local anaesthesia; MHRA, Medicines and Healthcare products Regulatory Agency; MOCA, Mechanochemical ablation; NHS, National Health Service; PE, Pulmonary embolism; QoL, Quality of life; R\&D, Research and Development; RCT, Randomised clinical trial; REC, Research Ethics Committee; RFA, Radiofrequency ablation; SAE, Serious adverse event; SAR, Serious adverse reaction; SF36, Short Form 36-item; SFJ, Saphenofemoral junction; SmPC, Summary of product characteristics; SPJ, Saphenopopliteal junction; STS, Sodium tetradecyl sulphate; SUSAR, Serious unexpected serious adverse reaction; SVI, Superficial venous insufficiency; TA, Tumescent anaesthesia; VAS, Visual analogue scale; VCSS, Venous Clinical Severity Score; VEINES-QOL/ Sym, VEnous INsufficiency Epidemiological and Economic Study to evaluate Quality of Life and Symptoms
\end{abstract}

\section{Background}

Varicose veins are a common disease worldwide, and in the United Kingdom, it affects approximately one-third of the population [1]. They are associated with symptoms causing pain and disability, soft tissue damage and venous ulcer, resulting in significant quality of life impairments [2] with consequent healthcare costs [3].

Endovenous thermal ablation (EVTA) techniques, such as endovenous laser ablation (EVLA), are now widely accepted and they are recognised as the first-line treatment for truncal varicose veins or superficial venous insufficiency (SVI) $[4,5]$. This approach has been shown to allow an enhanced recovery, with less pain and disability, resulting in superior early quality of life (QoL) when compared with surgical ligation and stripping, and improved efficacy when compared with foam sclerotherapy [6-11]. However, thermal ablation techniques carry the risk of damaging perivenous soft tissue and/or nerves. Thus, patients are treated with tumescent anaesthesia (TA), which requires multiple injections in order to infiltrate around the entire length of the target vein. Our Public and Patient Involvement (PPI) group and patients report that one of the major burdens associated with endovenous thermal ablation is the discomfort during injections of TA. Some patients still experience weeks of post-procedural pain despite the use of TA [12].

Mechanochemical ablation (MOCA), using the ClariVein device (Vascular Insights, Madison, CT, United States) is a newer treatment aiming to match the efficacy of thermal ablation whilst using a gentle sclerotherapy technique, with no requirement for TA. A catheter placed within the vein deploys a rotating hollow wire which causes physical damage to the endothelium and the vein goes into spasm. At the same time, a sclerosing agent is injected through the hollow wire into the vein, which results in protein denaturation, endothelial destruction and endoluminal fibrosis [13, 14]. Since no heat is involved and TA is made redundant, thermal-related complications such as pain, haematoma, induration and nerve injury could be reduced.

In the first human safety study, 30 great saphenous veins (GSVs) with primary SVI in 29 patients were treated with MOCA using sodium tetradecyl sulphate (STS) (Sotradecol). At 6 months [15] and at 2 years [16], the technical efficacy was $97 \%$ (29 of 30) and $96 \%$ (27 of 28), respectively. Several early case series have reported technical efficacy, with occlusion rates varying from 87 to $97 \%$ [17-20]. There were no serious adverse events such as pulmonary embolism (PE), deep vein thrombosis (DVT) or nerve injury observed in all previous studies. Furthermore, prospective observational cohort studies suggest MOCA was associated with significantly less procedure times, lower post-procedural pain and faster recovery than endovenous thermal ablation techniques [21, 22]. To date, no studies have been performed to compare MOCA with EVLA in the treatment of SVI. The current study has been designed to compare MOCA with EVLA in the treatment of SVI in a randomised controlled trial $(\mathrm{RCT})$.

\section{Methods/Design \\ Study objectives}

The aim of this randomised clinical trial is to establish whether MOCA is superior to the current first-line treatment, such as EVLA. The two main hypotheses are that MOCA may cause less initial pain and disability, allowing a more acceptable treatment with an enhanced recovery. The second hypothesis is that this may come at a cost of decreased efficacy, which may lead to increased recurrence and affect longer term quality of life, increasing the requirement of secondary procedures.

\section{Study design and setting}

This is a phase IV randomised clinical trial in the setting of a University Teaching Hospital, based in United Kingdom, offering tertiary vascular surgery services. 


\section{Inclusion criteria}

The inclusion criteria are: aged 18 or over; primary symptomatic SVI; reflux greater than 0.5 seconds in the saphenous veins; clinical grades $\mathrm{C} 2$ to $\mathrm{C} 6$; proposed treatment lengths of at least $10 \mathrm{~cm}$; treatment with either EVLA or MOCA is technically feasible; and written informed consent.

\section{Exclusion criteria}

The exclusion criteria are: one of the treatments is thought to be preferable by either the patient or an experienced endovenous specialist; known allergy to medications or dressings used in the treatment; known right to left circulatory shunt; evidence of acute deep vein thrombosis or complete ipsilateral occlusion; pelvic vein insufficiency; active or recent thrombophlebitis (within 6 weeks); impalpable foot pulses with the Ankle Brachial Pressure Index of less than 0.8; pregnancy or breast feeding; active malignancy; immobility; involvement in other Clinical Trials of an Investigational Medicinal Product (CTIMP) within the last 6 months; and unwell or inability to comply with the requirements for follow-up visits.

\section{Treatment}

All varicose vein procedures were carried out under ultrasound guidance and local anaesthesia (LA) by vascular surgeons who were experienced in both techniques of endovenous ablation. No preoperative analgesia or sedation is used. Initial vein access was performed under ultrasound guidance after injection of local anaesthetic (1\% lidocaine). With the patient in the reverse Trendelenburg position, the target vein will be cannulated percutaneously under duplex ultrasound scan (DUS) at the lowest point of demonstrable reflux using a 5-Fr sheath and dilator, followed by a 0.018 " guidewire. The total length of truncal vein treated will be documented.

For EVLA, the dilator and guidewire will be exchanged for a larger 0.035" wire using the Seldinger technique. The treatment sheath is then introduced and its tip accurately positioned at the saphenofemoral junction (SFJ) or saphenopopliteal junction (SPJ) (as appropriate) under DUS, aiming for a flush occlusion. The patient is then put placed in the horizontal position, and perivenous tumescent anaesthesia (TA) is infiltrated around the entire length of the target vein under DUS using a spinal needle and a pedal-operated peristaltic pump. The constituents of TA are $100 \mathrm{ml}$ of $1 \%$ lidocaine with $1: 200,000$ epinephrine in $900 \mathrm{ml}$ of $0.9 \%$ sodium chloride, which is buffered to $\mathrm{pH} 7.4$ with $10 \mathrm{ml}$ of $8.4 \%$ sodium bicarbonate. A sterile NeverTouch Gold-Tip laser fibre is introduced and locks with the treatment sheath. Endovenous laser energy is delivered using the VenaCure $1470 \mathrm{~nm}$ laser generator
(Angiodynamics, Waterlooville, UK) set at a continuous power delivery of $10 \mathrm{~W}$.

During MOCA, the ClariVein catheter (Vascular Insights, Glasgow, UK) will be inserted, aided by the 0.018" guidewire, and its tip is placed near the SFJ and SPJ, as appropriate under DUS. The catheter is connected to a $9 \mathrm{~V}$ battery-motorised handle unit that controls wire rotation, and the patient is treated in a horizontal position. The wire is activated for 10 seconds in order to induce vasospasm, and the device is withdrawn with a speed of approximately 7 seconds per centimetre, while the sclerosant is continuously injected. The sclerosing agent will be sodium tetradecyl sulphate (STS), also known as STD injection and marketed as Fibrovein $3 \%, 1 \%, 0.5 \%$ and $0.2 \%$ Solution for Injection (STD Pharmaceutical Products, Hereford, UK). The concentration used will be $1.5 \%$, made from $2 \mathrm{ml}$ ampoule of $3 \%$ STS with equal measures of $2 \mathrm{ml}$ sterile water (water for injection). Alternatively, diluting with $2 \mathrm{ml}$ of $0.9 \%$ normal saline is also satisfactory. The concentration and volume used will be clearly documented.

If required, concomitant ambulatory phlebectomy of the varicose tributaries will then be performed using the standard Oesch hook technique through 1-2 mm stab incisions. The same TA will be infiltrated around any tributaries to be treated. The phlebectomy sites will be dressed with Steri-Strip $^{\mathrm{Tm}}$ (3 M, St Paul, MN, USA), cotton wool, gauze and elastic compression dressings applied from foot to knee or groin, as appropriate. This will be exchanged for a thigh-length anti-thromboembolism stocking for 6 days after 24 hours. Patients will be advised to immediately mobilise within their comfort level, and to return to their normal activities and work as soon as they feel able to. Analgesia will not be routinely prescribed.

\section{Primary outcomes}

The joint primary outcomes will assess the hypothesised advantages and disadvantages of MOCA when compared with EVLA. The first will be patient-reported intraprocedural pain measured on a standardised $100-\mathrm{mm}$ visual analogue scale (VAS). The second will be technical efficacy at 1 year, with successful procedure defined as complete occlusion of the target vein segment. This will be assessed using duplex ultrasound.

\section{Secondary outcomes}

The secondary outcomes are procedure time, postprocedural pain, analgesia use, clinical severity, generic and disease-specific quality of life (QoL), bruising, complications, satisfaction, cosmesis, time taken to return to daily activities and/or work, and cost-effectiveness analysis following EVLA or MOCA.

The procedural time is from time of starting preparation to time of finishing dressing. Post-procedural pain 
will be recorded on a 100-mm VAS during the first week after treatment. The type and daily dosage of any analgesia taken by patients will also be recorded in a diary for the first week. Bruising, satisfaction with treatment and cosmesis will be recorded on a separate 100mm VAS.

Major complications include deep vein thrombosis, pulmonary embolus, stroke, loss of vision, damage to major artery, vein and/or motor nerve. Minor complications include superficial thrombophlebitis, numbness, ecchymosis, persistent tenderness, lumpiness, skin staining and wound infection.

Disease-specific QoL is evaluated using the Aberdeen Varicose Vein Questionnaire (AVVQ), the Chronic Venous disease quality of life Questionnaire (CIVIQ-20) and the VEnous INsufficiency Epidemiological and Economic Study to evaluate Quality of Life and Symptoms (VEINESQOL/Sym). AVVQ is a validated instrument that reflects the health status impairment associated specifically with venous disease [23]. CIVIQ-20 was developed more recently and it is designed to be more patient-centred [24]. VEINES-QOL/Sym is a 26-item patient-reported diseasespecific questionnaire to evaluate the QoL and symptoms across the full spectrum of conditions related to chronic venous disorders of the leg [25].

Generic QoL is assessed using the Short Form 36-item (SF-36) and EuroQol 5-domain utility index (EQ5D). The SF-36 is a multidimensional measurement of general health, which yields eight domains of functional health and well-being scores [26-28]. EQ5D is an index scale mapping three available responses to five domain questions [29], which is transformed using the UK time trade-off tariffs into a global single index scale [30]. Both have undergone extensive testing of validity and reliability, including in the context of venous insufficiency and treatment [31-33].

Two validated objective measures will be used to assess and classify the severity of disease. The first is the Clinical severity, Etiology, Anatomy and Pathophysiology (CEAP) classification, which classifies severity into six grades. The second is the Venous Clinical Severity Score (VCSS), which grades three components from $0-3$ with increasing severity, and then summates them into a single score. Clinical success is the objective improvement of clinical outcome after treatment.

Post-procedural DUS will assess the treatment efficacy [34]. Initial treatment success will be defined as complete target vein occlusion at 1 and/or 6 weeks. Anything else will be regarded as a technical failure. Recanalisation will be assessed at 52 weeks and is defined as blood flow within the target vein which has been treated. This will be broken down into partial $<25 \%$ or full $\geq 25 \%$ of the length of the treated vein. Residual disease is regarded as any reflux which was also present at baseline, but not a target for ablation. Disease progression will be defined as any reflux within a vein which was not present on baseline assessment. In the presence of clinical recurrence, DUS will be used to map out the pattern of the recurrence as this may give insight into techniques to avoid further recurrence and aid understanding of how recurrence comes about after these novel treatments. Post-procedural DUS will also look for evidence of complications such as heatinduced thrombosis, deep vein thrombosis, haematoma and superficial thrombophlebitis.

\section{Trial timescales}

At the current rate of patients presenting to the unit, relatively wide inclusion criteria and experience from previous trials of this nature, it is anticipated that the recruitment and treatment phase would be complete within approximately 18 months. The primary endpoint is measured at 1 year and therefore completion of data collection is anticipated in approximately 30 months (Fig. 1).

\section{Sample size calculation}

The power calculation is based upon the joint primary endpoints with $90 \%$ power and $5 \%$ significance. A published comparison of MOCA and radiofrequency thermal ablation found a reduction in intra-procedural pain from $35 \mathrm{~mm}$ to $19 \mathrm{~mm}$ on a $100-\mathrm{mm}$ visual analogue scale (VAS) with a standard deviation of $20 \mathrm{~mm}$ [35]. This gives a required sample size of 33 patients per group or 73 in the trial including $10 \%$ loss to follow-up. This difference is comparable with differences in patient-reported pain VAS, which was previously found to be associated with a difference in physical domains of quality of life and associated with changes in recovery time [8], and therefore can be judged to be clinically significant. Previous comparisons of radiofrequency ablation (RFA) with EVLA have suggested that EVLA is associated with more post-procedural pain [12, 36-38]. However older laser delivery technology (bare-tip fibres), shorter wavelength of laser (810-980 nm), additional treatment to truncal varicose vein with the allocated treatment at the same sitting, and general anaesthesia (GA) were involved; thus their findings may not be applicable today. Furthermore, no study has shown any significant benefit in quality of life relating to the reported differences.

The same study [35] reported complete target vein occlusion in $83 \%$ of patients following MOCA at 1 month. Our previous RCT found target vein occlusion in $99 \%$ at 1 year following EVLA [7]. A difference of this magnitude is likely to be clinically significant and have implications towards the long-term durability of the procedure, affecting effectiveness and cost-effectiveness. The required sample size to detect such a difference, if it exists, would be 62 


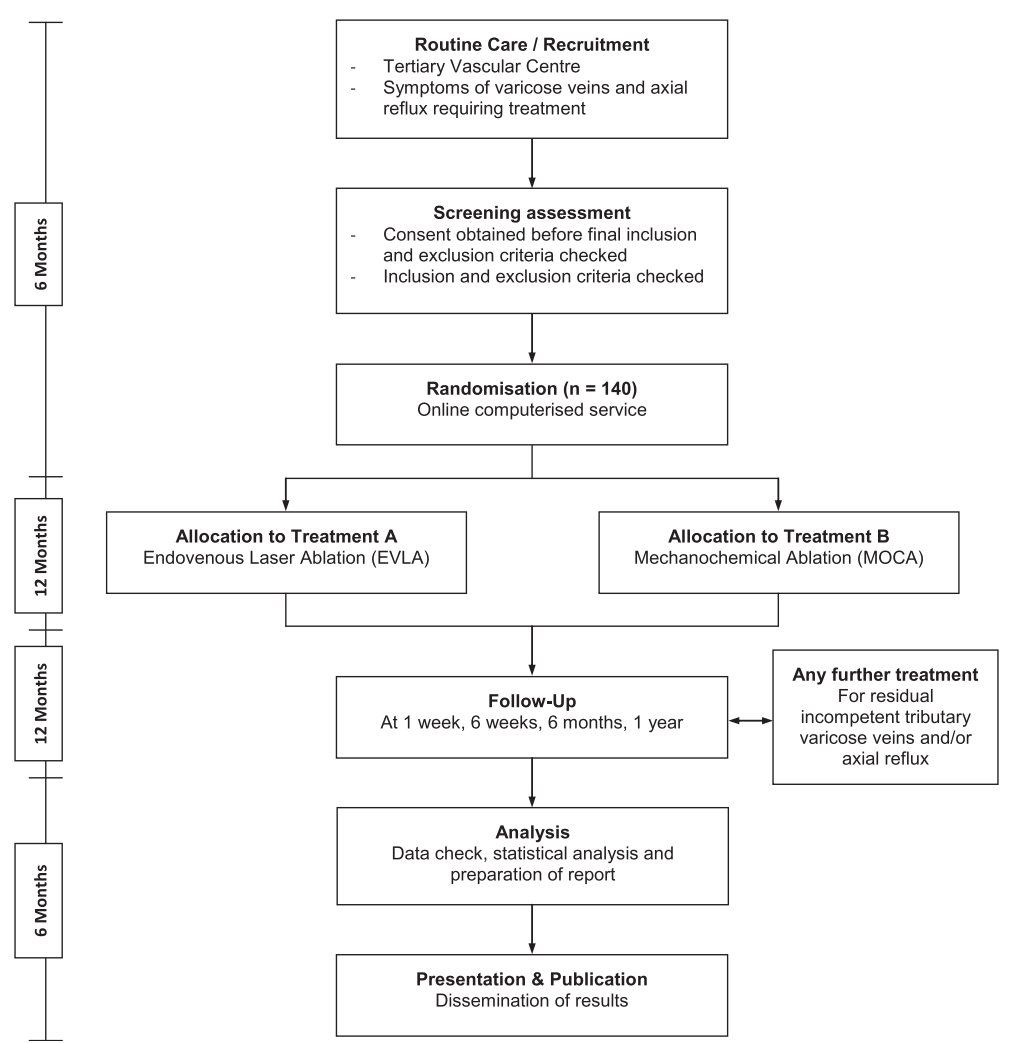

Fig. 1 Study flow chart. The total target sample size is 140, which will be randomised to endovenous laser ablation $(n=70)$ or mechanochemical ablation $(n=70)$

per group or 137 including $10 \%$ loss to follow-up. Taking this into consideration, the target sample size is 140 .

\section{Recruitment}

Each patient referred to the vascular surgery services with symptomatic SVI is assessed. Patients who potentially meet the inclusion criteria will be made aware of this research study and provided with the appropriate information, including the Patient Information Sheet. Patients expressing an interest in participation will be offered an appointment for a screening visit with a study investigator. At the screening appointment, the medical history and examination will be reviewed, followed by a detailed duplex ultrasound examination according to a set protocol based upon international consensus $[39,40]$. If the potential participant meets the required inclusion criteria without any exclusion criteria, subsequent discussion of the study will take place in full (Fig. 1). If the potential participant meets the inclusion criteria for the study and is willing and able to proceed to enrolment in the trial, they will then be consented using a standardised Patient Consent Form. The Co-Investigators and Principle Investigator will obtain informed consent. Here the potential participant will be fully briefed on the trial process, the treatments, follow-up, time commitments and that this will be more detailed than regular follow-up within the non-trial setting.

To ensure confidentiality and to adhere to the Caldicott and Data Protection guidance, the participant will be assigned a unique study number for identification purposes; this will not allow identification of the study arm or any demographic information. No information identifying individuals including the study ID number will be made available to anyone outside the research group. A letter will be sent to the participant's General Practitioner to inform them of their enrolment into the study and its details.

\section{Randomisation}

Consented participants will be allocated to one of the two parallel treatments groups by equal randomisation (Fig. 1), which will be conducted using an online computerised service (Sealed Envelope, London, UK).

\section{Blinding}

Due to the nature of the procedures involved it will not be possible to blind the participant or clinical team as to which group the participant is allocated. Where possible, assessor- reported outcomes will be performed by an independent assessor who is blinded to the treatment 
allocation. Bias in other outcomes will be limited by the use of predetermined standardised objective measurements, standardised protocols, and the extensive use of patient-reported outcomes measures.

\section{Data collection}

Data for all outcomes from each participant's visits will be assimilated into the participants' unique case report form (CRF) and anonymised into a Microsoft Access database to allow further analyses, and monitored by the Research \& Development (R\&D) department.

\section{Study visits}

Baseline measurements will be collected from all participants once consent is obtained and prior to randomisation. Study measurements will be taken on the day of treatment and on follow-up at 1 week, 6 weeks, 6 months and 1 year. Clinical assessment, duplex ultrasound and questionnaires are completed at these follow-up time points (Table 1).

\section{Statistical analysis}

Results will be evaluated based on an intention-to-treat analysis. Continuous data will first be tested for normality. Normally distributed data will be presented as mean and standard deviation, and hypothesis testing performed with paired and unpaired $t$ tests, using two-sided significance tests with a $5 \%$ significance threshold. If the data is not normally distributed, median and interquartile range values will be presented, with analysis using the Mann-Whitney $U$ test for unrelated samples and Wilcoxon signed rank test for paired data. Categorical data will be analysed by means of $X^{2}$ test or, if necessary, Fisher's exact test. Occlusion rates will be presented as Kaplan-Meier curves, including censoring.

\section{Monitoring, safety and quality control}

This study will be monitored in accordance with the Research \& Development (R\&D) Department's standard operating procedures to ensure compliance with the International Conference on Harmonisation, Good Clinical Practice and the Research Governance Framework 2005.

Table 1 Schedule of assessments

\begin{tabular}{|c|c|c|c|c|c|c|}
\hline Visits & 1 & 2 & 3 & 4 & 5 & 6 \\
\hline Procedures & $\begin{array}{l}\text { Screening, eligibility, baseline } \\
\text { assessment and randomisation }\end{array}$ & Treatment & 1-week follow-up & 6-week follow-up & 6-month follow-up & 1-year follow-up \\
\hline Medication history & $x$ & $x$ & $x$ & $x$ & $x$ & $x$ \\
\hline Medications & $x$ & $x$ & $x$ & $x$ & $x$ & $x$ \\
\hline Physical examination & $x$ & $x$ & $x$ & $x$ & $x$ & $x$ \\
\hline NS-SEC & $x$ & & & & & \\
\hline Employment status & $x$ & & & & & \\
\hline Informed consent & $x$ & & & & & \\
\hline CEAP & $x$ & & $x$ & $x$ & $x$ & $x$ \\
\hline VCSS & $x$ & & $x$ & $x$ & $x$ & $x$ \\
\hline AWQ & $x$ & & $x$ & $x$ & $x$ & $x$ \\
\hline CIVIQ-20 & $x$ & & $x$ & $x$ & $x$ & $x$ \\
\hline VEINES-QOL/Sym & $x$ & & $x$ & $x$ & $x$ & $x$ \\
\hline SF-36 & $x$ & & $x$ & $x$ & $x$ & $x$ \\
\hline EQ5D & $x$ & & $x$ & $x$ & $x$ & $x$ \\
\hline DUS & $x$ & & $x$ & $x$ & $x$ & $x$ \\
\hline Pain VAS & & $x$ & $x$ & $x$ & $x$ & $x$ \\
\hline Analgesia diary & & $x$ & $x$ & $x$ & $x$ & $x$ \\
\hline Satisfaction VAS & & & $x$ & $x$ & $x$ & $x$ \\
\hline Cosmesis VAS & & & $x$ & $x$ & $x$ & $x$ \\
\hline Recovery time & & & $x$ & $x$ & $x$ & $x$ \\
\hline Complications & & & $x$ & $x$ & $x$ & $x$ \\
\hline
\end{tabular}

NS-SEC National Statistics Socio-Economic Classification, CEAP Clinical severity, Etiology, Anatomy and Pathophysiology, VCSS Venous Clinical Severity Score, AVVQ Aberdeen Varicose Vein Questionnaire, CIVIQ-20 Chronic Venous Insufficiency Questionnaire, VEINES-QOL/Sym VEnous INsufficiency Epidemiological and Economic Study to evaluate Quality of Life and Symptoms, SF-36 Short Form 36-item, EQ5D EuroQol 5-domain utility index, DUS duplex ultrasound, VAS visual analogue scale 
Adverse events (AE) are defined as any untoward medical occurrence in a subject whom a medicinal product has been administered, or a procedure performed, as part of a research study, including occurrences which are not necessarily caused by or related to that investigational medicinal product (IMP). Serious adverse event (SAE) is any event if it results in death, is life-threatening, requires hospitalisation or prolongation of existing hospitalisation, results in significant or persisting disability or incapacity, and is a congenital anomaly or birth defect. Adverse reaction (AR) is any untoward or unintended response in a subjection to an IMP. Serious adverse reaction (SAR) is a serious event which is suspected (possibly, probably or definitely) to be related to an IMP and expected for the IMP. Suspected unexpected serious adverse reaction (SUSAR) is a serious event which is suspected (possibly, probably or definitely) to be related to an IMP and unexpected for the IMP, i.e. not previously documented in any of the IMP information (SmPC) or protocol.

The AE reporting period for this trial begins as soon as patients have consented to the trial and ends 30 days after the patient's treatment visit. The health status of subjects will be checked at each study visit. The investigator will record all directly observed $\mathrm{AE}$ and all $\mathrm{AE}$ spontaneously reported by the trial subject. A pre-existing condition is a disorder present prior to the patient entering the trial and does not need to be reported as an $\mathrm{AE}$ unless the condition worsens or episodes increase in frequency during the $\mathrm{AE}$ reporting period. All $\mathrm{AE}$ will be followed up by investigators until the event has resolved or a decision has been taken for no further follow-up. All AE (serious and nonserious) will be recorded by the investigator in the case report forms. A description of the event, including start date, end date, action taken and the outcome will be provided.

Investigators will notify the R\&D Department of any SAE within 24 hours of becoming aware of the event. The R\&D Department will report fatal or life-threatening SUSARs to the Medicines and Healthcare products Regulatory Agency (MHRA) within 7 days and follow-up information in a further 8 days. The R\&D Department will send all other SUSAR reports to the MHRA within a maximum of 15 days. The investigator will repot fatal or life-threatening SUSARs to the Research Ethics Committee (REC) within 7 days and follow-up information within a further 8 days. The investigator will send all other SUSAR reports to the REC within a maximum of 15 days. The investigators will submit a Development Safety Update Report (DSUR) to the MHRA 12 months after the date of the MHRA clinical trial authorisation and thereafter until the end of the study according to the MHRA website.

Participants have access to information on complaints procedure and for obtaining compensation and treatment following harm through negligence or non-negligence as a direct result of participating in the trial.
The end of trial is defined as the last visit of the last subject completing their 1-year follow-up assessment. In accordance with Trust policy all personal and/or sensitive personal data (as defined by the Data Protection Act 1998) will be securely destroyed at the conclusion of the research. Non-identifiable data and other records not containing person-identifiable data may be retained for a longer period at the discretion of the Principle Investigator. These will be stored appropriately to ensure its integrity, confidentiality and accessibility.

\section{Discussion}

Endovenous techniques have revolutionised the treatment of truncal varicose veins, and endovenous thermal ablation has become the recommended first-line treatment method [4], achieving occlusion rates of greater than $90 \%$ [11]. However, the search for the optimum treatment method is still ongoing and recent emphasis has focused on improving outcomes such as intra- and post-procedural pain, and reducing thermal-related injury and complications. A potential solution to the problems raised by endovenous thermal ablation is the use of newer non-thermal and nontumescent anaesthesia treatment methods such as MOCA.

The aim of the present randomised clinical trial is twofold. The first hypothesis is that MOCA may cause less initial pain and disability, allowing a more acceptable treatment with an enhanced recovery. The second hypothesis is that this may come at a cost of decreased efficacy, which may lead to increased recurrence and affect longer term QoL, increasing the requirement for secondary procedures. In order to have sufficient power, the trial was designed using a non-inferiority principle.

In conclusion, the LAMA trial is a randomised controlled trial that aims for reduction in intra- and postprocedural pain after MOCA compared with EVLA, with a similar clinical success and technical efficacy.

\section{Trial status}

The LAMA trial began recruitment of participants in June 2015. By the end of December 2015, 75 patients had provided written informed consent and were subsequently randomised to either treatment.

\author{
Acknowledgements \\ Not applicable. \\ Funding \\ The authors receive no additional funding for these research activities.
}

\section{Authors' contributions}

CCML drafted the manuscript. DC and TW co-authored the writing of the manuscript. CCML, DC and ICC contributed substantially to the design of the study. DC and ICC performed sample size calculation. CCML designed the computerised database. DC, TW and ICC contributed to the scientific accuracy of the manuscript. All authors critically revised the manuscript, and read and approved the final manuscript. 


\section{Authors' information}

CCML is a Clinical Research Fellow in Vascular Surgery.

DC is a NIHR Academic Clinical Lecturer in Vascular Surgery.

TW is a NIHR Academic Clinical Lecturer in Vascular Surgery.

ICC is a Consultant in Vascular Surgery and Professor of Surgery at Hull York Medical School.

\section{Competing interests}

The authors declare that they have no competing interests.

\section{Consent for publication}

The Participant Information Sheet and Participant Consent Form, which have been approved by the REC, provide information that the results will be submitted for publication in a scientific journal and a final report written. Participants will not be identified in any reports or publications. The results will be disseminated via presentations to societies and publication in peer-reviewed journals. Co-authorship will be assigned according to the "Recommendations for the Conduct, Reporting, Editing, and Publication of Scholarly Work in Medical Journals, Updated December 2015" of the International Committee of Medical Journal Editors.

\section{Ethics approval and consent to participate}

This study is conducted in accordance with the principles of the Declaration of Helsinki, the Medicine for Human Use Regulations 2004 and Amendment Regulations 2006 and subsequent amendments, the Good Clinical Practice guidelines, and the Research Governance Framework for Health and Social Care 2005. The Research Protocol, Participant Information Sheet, and Participant Consent Form have been approved by the National Research Ethics Service Committee Yorkshire \& The Humber - Sheffield (15/YH/0207), the Medicines and Healthcare Products Regulatory Agency (21411/0250/0010001), and the Hull and East Yorkshire Hospitals National Health Service (NHS) Trust Research \& Development Department (R1788). If the potential participant meets the inclusion criteria for the study and is willing and able to proceed to enrolment in the trial, the Co-Investigators and Principle Investigator will obtain informed consent. The potential participant will be fully briefed on the trial process, the treatments, follow-up time commitments and that this will be more detailed than regular followup within the non-trial setting. A valid, dated and signed Participant Consent Form is required to take part in the study.

\section{Name and contact of trial sponsor}

Hull and East Yorkshire Hospitals NHS Trust Research \& Development Department James Illingworth

Email: james.illingworth@hey.nhs.uk

Office 14, 2nd Floor Daisy Building, Castle Hill Hospital, Cottingham, East Yorkshire, HU16 5JQ

\section{Received: 29 March 2016 Accepted: 21 July 2016}

Published online: 24 August 2016

\section{References}

1. Evans CJ, Fowkes FGR, Ruckley CV, Lee AJ. Prevalence of varicose veins and chronic venous insufficiency in men and women in the general population: Edinburgh vein study. J Epidemiol Community Health. 1999;53:149-53.

2. Shepherd AC, Gohel MS, Lim CS, Davies AH. A study to compare diseasespecific quality of life with clinical anatomical and hemodynamic assessments in patients with varicose veins. J Vasc Surg. 2011;53:374-82.

3. Van den Oever R, Hepp B, Debbaut B, Simon I. Socio-economic impact of chronic venous insufficiency. An underestimated public health problem. Int Angiol. 1998;17:161-7.

4. National Institute for Health and Care Excellence. Varicose veins in the legs: the diagnosis and management of varicose veins. Clinical guideline 168. 2013. https://www.nice.org.uk/guidance/cg168. Accessed 28 Apr 2016.

5. Gloviczki P, Comerota AJ, Dalsing MC, Eklof BG, Gillespie DL, Gloviczki ML, Lohr JM, McLafferty RB, Meissner MH, Murad MH, Padberg FT, Pappas PJ, Passman MA, Raffetto JD, Vasquez MA, Wakefield TW, Society for Vascular Surgery, American Venous Forum. The care of patients with varicose veins and associated chronic venous diseases: clinical practice guidelines of the Society for Vascular Surgery and the American Venous Forum. J Vasc Surg. 2011;53:2S-48
6. Brittenden J, Cotton SC, Elders A, Ramsay CR, Norrie J, Burr J, Campbell B, Bachoo P, Chetter I, Gough M, Earnshaw J, Lees T, Scott J, Baker SA, Francis J, Tassie E, Scotland G, Wileman S, Campbell MK. A randomized trial comparing treatments for varicose veins. N Engl J Med. 2014;371:1218-27.

7. Carradice D, Mekako Al, Mazari FA, Samuel N, Hatfield J, Chetter IC. Clinical and technical outcomes from a randomized clinical trial of endovenous laser ablation compared with conventional surgery for great saphenous varicose veins. Br J Surg. 2011;98:1117-23.

8. Carradice D, Mekako Al, Mazari FA, Samuel N, Hatfield J, Chetter IC. Randomized clinical trial of endovenous laser ablation compared with conventional surgery for great saphenous varicose veins. Br J Surg. 2011;98:501-10.

9. Samuel N, Carradice D, Wallace T, Mekako A, Hatfield J, Chetter I. Randomized clinical trial of endovenous laser ablation versus conventional surgery for small saphenous varicose veins. Ann Surg. 2013;257:419-26.

10. Nandhra S, El-Sheikha J, Carradice D, Wallace T, Souroullas P, Samuel N, Smith G, Chetter IC. A randomised clinical trial of endovenous laser ablation versus conventional surgery for small saphenous varicose veins. J Vasc Surg. 2015;61:741-6.

11. Rasmussen LH, Lawaetz M, Bjoem L, Vennits B, Blemings A, Eklof B. Randomized clinical trial comparing endovenous laser ablation, radiofrequency ablation, foam sclerotherapy and surgical stripping for great saphenous varicose veins. Br J Surg. 2011;98:1079-87.

12. Shepherd AC, Gohel MS, Brown LC, Metcalfe MJ, Hamish M, Davies AH. Randomized clinical trial of VNUS Closure FAST radiofrequency ablation versus laser for varicose veins. Br J Surg. 2010;97:810-8.

13. Kendler M, Averbeck M, Simon JC, Ziemer M. Histology of saphenous veins after treatment with the ClariVein device - an ex-vivo experiment. J Dtsch Dermatol Ges. 2013;11:348-52.

14. van Eekeren RR, Hillebrands JL, van der Sloot K, de Vries JP, Zeebregts CJ, Reijnen MM. Histological observations one year after mechanochemical endovenous ablation of the great saphenous vein. J Endovasc Ther. 2014; 21:429-33.

15. Elias S, Raines JK. Mechanochemical tumescentless endovenous ablation: final results of the initial clinical trial. Phlebology. 2012;27:67-72.

16. Elias S, Lam YL, Wittens $\mathrm{CH}$. Mechanochemical ablation: status and results. Phlebology. 2013;28 Suppl 1:10-4.

17. van Eekeren RR, Boersma D, Elias S, Holewijn S, Werson DA, de Vries JP, Reijnen MM. Endovenous mechanochemical ablation of great saphenous vein incompetence using the ClariVein device: a safety study. J Endovasc Ther. 2011;18:328-34.

18. Boersma D, van Eekeren RR, Werson DA, van der Waal RI, Reijnen MM, de Vries JP. Mechanochemical endovenous ablation of small saphenous vein insufficiency using the Clarivein device: one-year results of a prospective series. Eur J Vasc Endovasc Surg. 2013;45:299-303.

19. Bishawi M, Bernstein R, Boter M, Draughn D, Gould CF, Hamilton C, Koziarski J. Mechanochemical ablation in patients with chronic venous disease: a prospective multicenter report. Phlebology. 2014;29:397-400.

20. Kim PS, Bishawi M, Draughn D, Boter M, Gould C, Koziarski J, Bernstein R, Hamilton R. Mechanochemical ablation for symptomatic great saphenous vein reflux: a two-year follow-up. Phlebology. 2016 [Epub ahead of print].

21. van Eekeren RR, Boersma D, Konijn V, de Vries JP, Reijnen MM. Postoperative pain and early quality of life after radiofrequency ablation and mechanochemical endovenous ablation of incompetent great saphenous veins. J Vasc Surg. 2013;57:445-50.

22. Vun SV, Rashid ST, Blest NC, Spark Jl. Lower pain and faster treatment with mechanico-chemical endovenous ablation using ClariVein . Phlebology. 2015;30:688-92.

23. Garratt AM, Macdonald LM, Ruta DA, Russell IT, Buckingham JK, Krukowski $\mathrm{ZH}$. Towards measurement of outcome for patients with varicose veins. Qual Health Care. 1993;2:5-10.

24. Launois R, Mansilha A, Jantet $G$. International psychometric validation of the Chronic Venous Disease quality of life Questionnaire (CIVIQ-20). Euro J Vasc Endovasc Surg. 2010;40:783-9.

25. Lamping DL, Schroter S, Kurz X, Kahn SR, Abenhaim L. Evaluation of outcomes in chronic venous disorders of the leg: development of a scientifically rigorous, patientreported measure of symptoms and quality of life. J Vasc Surg. 2003;37:410-9.

26. Ware Jr JE, Sherbourne CD. The MOS 36-item short-form health survey (SF36): I. Conceptual framework and item selection. Med Care. 1992;30:473-83.

27. McHorney CA, Ware Jr JE, Raczek AE. The MOS 36-item short-form health survey (SF-36): II. Psychometric and clinical tests of validity in measuring physical and mental health constructs. Med Care. 1993;31:247-63. 
28. McHorney CA, Ware Jr JE, Lu JF, Sherbourne CD. The MOS 36-item shortform health survey (SF-36): III. Tests of data quality, scaling assumptions, and reliability across diverse patient groups. Med Care. 1994;32:40-66.

29. The EuroQol Group. EuroQol - a new facility for the measurement of health-related quality of life. Health Policy. 1990;16:199-208.

30. Dolan P, Gudex C, Kind P, Williams A. The time trade-off method: results from a general population study. Health Econ. 1996;5:141-54.

31. Garratt AM, Ruta DA, Abdalla MI, Russell IT. Responsiveness of the SF-36 and a condition-specific measure of health for patients with varicose veins. Qual Life Res. 1996;5:223-34.

32. Smith JJ, Garratt AM, Guest M, Greenhalgh RM, Davies AH. Evaluating and improving health-related quality of life in patients with varicose veins. J Vasc Surg. 1999;30:710-9.

33. Michaels JA, Campbell WB, Brazier JE, Maclntyre JB, Palfreyman SJ, Ratcliffe J, Rigby K. Randomised clinical trial, observational study and assessment of cost-effectiveness of the treatment of varicose veins (REACTIV trial). Health Technol Assess. 2006;10(13):1-196.

34. De Maeseneer M, Pichot O, Cavezzi A, Earnshaw J, van Rij A, Lurie F, Smith PC. Union Internationale de Phlebologie. Duplex ultrasound investigation of the veins of the lower limbs after treatment for varicose veins - UIP consensus document. Eur J Vasc Endovasc Surg. 2011;42:89-102.

35. Bootun R, Lane T, Dharmarajah B, Lim CS, Najem M, Renton S, Sritharan K, Davies AH. Intra-procedural pain score in a randomised controlled trial comparing mechanochemical ablation to radiofrequency ablation: the Multicentre Venefit ${ }^{\mathrm{TM}}$ versus ClariVein ${ }^{\circ}$ for varicose veins trial. Phlebology. 2016;31:61-5.

36. Almieda J, Kaufman J, Gockeritz O, Chopra P, Evans MT, Hoheim DF, Makhoul RG, Richards T, Wenzel C, Raines JK. Radiofrequency endovenous ClosureFAST versus laser ablation for the treatment of great saphenous reflux: a multicenter, single-blinded, randomised study (RECOVERY study). J Vasc Interv Radiol. 2009;20:752-9.

37. Goode SD, Chowdhury A, Crockett M, Beech A, Simpson R, Richards T, Braithwaite BD. Laser and radiofrequency ablation study (LARA study): a randomised study comparing radiofrequency ablation and endovenous laser ablation (810 nm). Eur J Vasc Endovasc Surg. 2010;40:246-53.

38. Nordon IM, Hinchliffe RJ, Brar R, Moxey P, Black SA, Thompson MM, Loftus IM. A prospective double-blind randomized controlled trial of radiofrequency versus laser treatment of the great saphenous vein in patients with varicose veins. Ann Surg. 2011;254:876-81.

39. Coleridge-Smith P, Labropoulos N, Partsch H, Myers K, Nicolaides A, Cavezzi A. Duplex ultrasound investigation of the veins in chronic venous disease of the lower limbs - UIP consensus document. Part I. Basic principles. Eur J Vasc Endovasc Surg. 2006;31:83-92.

40. Cavezzi A, Labropoulos N, Partsch H, Ricci S, Caggiati A, Myers K, Nicolaides A, Smith PC, UIP. Duplex ultrasound investigation of the veins in the chronic venous disease of the lower limbs - UIP consensus document. Part II. Anatomy. Eur J Vasc Endovasc Surg. 2006;31:288-99.

\section{Submit your next manuscript to BioMed Central and we will help you at every step:}

- We accept pre-submission inquiries

- Our selector tool helps you to find the most relevant journal

- We provide round the clock customer support

- Convenient online submission

- Thorough peer review

- Inclusion in PubMed and all major indexing services

- Maximum visibility for your research

Submit your manuscript at www.biomedcentral.com/submit

C Biomed Central 